\title{
Identical Accumulation and Immobilization of Sulfated and Nonsulfated Nod Factors in Host and Nonhost Root Hair Cell Walls
}

\author{
Joachim Goedhart, ${ }^{1}$ Jean-Jacques Bono, ${ }^{2}$ Ton Bisseling, ${ }^{1}$ and Theodorus W. J. Gadella, Jr. ${ }^{1}$ \\ ${ }^{1}$ Laboratory for Molecular Biology, MicroSpectroscopy Center Wageningen, Department of Plant Sciences, Wageningen \\ University, Dreijenlaan 3, $6703 \mathrm{HA}$, Wageningen, The Netherlands; ${ }^{2}$ Signaux et Messages Cellulaires chez les Végétaux, \\ UMR CNRS-UPS 5546, Pôle de Biotechnologie Végétale, 24 chemin de Borde Rouge, BP 17 Auzeville, 31326 Castanet- \\ Tolosan, France.
}

Submitted: 31 March 2003. Accepted 19 June 2003.

\begin{abstract}
Nod factors are signaling molecules secreted by Rhizobium bacteria. These lipo-chitooligosaccharides (LCOs) are required for symbiosis with legumes and can elicit specific responses at subnanomolar concentrations on a compatible host. How plants perceive LCOs is unclear. In this study, using fluorescent Nod factor analogs, we investigated whether sulfated and nonsulfated Nod factors were bound and perceived differently by Medicago truncatula and Vicia sativa root hairs. The bioactivity of three novel sulfated fluorescent LCOs was tested in a root hair deformation assay on M. truncatula, showing bioactivity down to 0.1 to 1 nM. Fluorescence microscopy of plasmolyzed $M$. truncatula root hairs shows that sulfated fluorescent Nod factors accumulate in the cell wall of root hairs, whereas they are absent from the plasma membrane when applied at $10 \mathrm{nM}$. When the fluorescent Nod factor distribution in medium surrounding a root was studied, a sharp decrease in fluorescence close to the root hairs was observed, visualizing the remarkable capacity of root hairs to absorb Nod factors from the medium. Fluorescence correlation microscopy was used to study in detail the mobilities of sulfated and nonsulfated fluorescent Nod factors which are biologically active on $M$. truncatula and $V$. sativa, respectively. Remarkably, no difference between sulfated and nonsulfated Nod factors was observed: both hardly diffuse and strongly accumulate in root hair cell walls of both $M$. truncatula and $V$. sativa. The implications for the mode of Nod factor perception are discussed.
\end{abstract}

Additional keywords: BODIPY, chitinase, diffusion, FCM, flipflop.

Nod factors are signaling molecules produced by rhizobia and are required to establish symbiosis with a compatible legume (Heidstra and Bisseling 1996; Long 1996). Nod factors are lipochitooligosaccharides (LCOs) comprising a chitin backbone of three to five $\beta$-1,4-linked $N$-acetylglucosamine (GlcNAc) residues and an acyl chain which is $N$-linked to the nonreducing terminal sugar. Nod factors, either purified from rhizobial cultures

Corresponding author: T. W. J. Gadella, Jr., Telephone: +31 20525 6259; Fax: +31 20525 6271; E-mail: Gadella@science.uva.nl

Current address of J. Goedhart and T. W. J. Gadella, Jr.: Laboratory for Molecular Cytology, Swammerdam Institute for Life Sciences, University of Amsterdam, Kruislaan 316, 1098 SM Amsterdam. or chemically synthesized, elicit a wide variety of specific responses at picomolar concentrations on their compatible host plant (Downie and Walker 1999). These responses include ion fluxes (Felle et al. 1998), calcium spiking (Ehrhardt et al. 1996; Wais et al. 2000; Walker et al. 2000), and activation of phospholipases (Den Hartog et al. 2001; Pingret et al. 1998), all within ten minutes; followed by root hair deformation (Heidstra et al. 1994; Lerouge et al. 1990) and gene expression (Pingret et al. 1998) after a few hours. The chemical substitutions on the chitin backbone, which are characteristic of Nod factors produced by a given species of rhizobia, are the major determinants of host specificity. In addition, the structure of the acyl chain can modulate biological activity (Demont-Caulet et al. 1999; Goedhart et al. 1999; Spaink et al. 1991). Nod factors produced by Rhizobium leguminosarum bv. viciae have an acetate on the nonreducing GlcNAc as the only substitution on the chitin backbone (Spaink et al. 1991), whereas Sinorhizobium meliloti Nod factors differ mainly by the presence of a sulfate group on O-6 of the reducing GlcNAc (Lerouge et al. 1990). The sulfate group is essential for establishing symbiosis with host plants like Medicago truncatula and Medicago sativa but is not tolerated by Vicia sativa (Roche et al. 1991). As a consequence, mutation of the rhizobial $n o d H$ gene (encoding a sulfotransferase), responsible for the 6-O-sulfation of Nod factors, abolishes symbiosis with $M$. truncatula. However, at high concentrations, nonsulfated Nod factors also can trigger responses in M. truncatula (Oldroyd et al. 2001), possibly reflecting some redundant affinity for these Nod factors by the putative signaling receptor. Furthermore, the nodH mutant is able to nodulate the nonhost $V$. sativa, in contrast to wild-type $S$. meliloti bacteria (Roche et al. 1991). Hence, when these two groups of legume hosts are considered, the presence or absence of the sulfate group is the primary determinant of host specificity.

The high specificity and the low concentration at which Nod factors are active strongly suggest the involvement of a Nod factor receptor (Cullimore et al. 2001). To elucidate the signaling pathway induced by Nod factors, mutants have been characterized that are disturbed in an early Nod factor response (i.e., calcium spiking) (Wais et al. 2000; Walker et al. 2000). Recently, a receptor kinase essential for Nod factor signal transduction, termed nodulation receptor kinase (NORK), has been cloned (Endre et al. 2002; Stracke et al. 2002). This gene is shown to be present in several legume members, including M. truncatula. However, whether the extracellular leucine-rich repeat is able to interact directly or indirectly with Nod factors is unknown. 
Biochemical approaches have led to the isolation of several Nod factor binding activities (Bono et al. 1995; Etzler et al. 1999; Gressent et al. 1999; Niebel et al. 1997). Bono and coworkers have characterized two different binding activities in an extract from $M$. truncatula roots and from extracts of an $M$. varia cell culture (Bono et al. 1995; Gressent et al. 1999; Niebel et al. 1997). A low affinity binding site (NFBS1; Kd = $86 \mathrm{nM}$ ) found in the root extract from $M$. truncatula also was present in the nonlegume tomato (Bono et al. 1995). In addition to a low-affinity site, a high-affinity site was found in an $M$. varia cell culture extract (NFBS2; Kd $=4 \mathrm{nM}$ ), which was enriched in the microsomal fraction (Gressent et al. 1999). Strikingly, both binding activities do not discriminate between sulfated and nonsulfated Nod factors.

Another strategy in obtaining insight into the mechanism of Nod factor perception is to study the location and molecular behavior of Nod factors when added to roots. A prerequisite is the presence of a sensitive and specific label on the Nod factor, which is satisfied by using a fluorescent reporter group. The use of ligands tagged with a fluorescent group has been applied successfully for several decades for the study of ligand-binding to receptors in mammalian cell cultures (Shechter et al. 1978). A variety of dedicated fluorescence techniques can be employed for studying ligand-receptor interactions (Hovius et al. 2000). So far, only a limited number of fluorescently labeled ligands have been used for plant studies. Previously, we described the use of nonsulfated fluorescent Nod factor derivatives in the study of Nod factor perception in V. sativa (Gadella et al. 1997; Goedhart et al. 2000). We could show that Nod factors, when applied at low concentration, are located within the cell wall, and are absent from the plasma membrane (Goedhart et al. 2000). In the same study, fluorescence correlation microscopy (FCM) was used to monitor the diffusional characteristics of the fluorescent Nod factors on the root hairs of $V$. sativa. FCM measures intensity fluctuations originating from single fluorescent molecules moving across a stationary laser spot, which can be related to diffusion constants and particle numbers (Hess et al. 2002; Maiti et al. 1997). The FCM experiments revealed that the fluorescent Nod factors accumulate and are immobilized within root hair cell walls of $V$. sativa (Goedhart et al. 2000). Together, these results were surprising, because it has been shown that, in the presence of membrane-like structures, Nod factors insert their lipid tail into the hydrophobic core of the membrane, suggesting association with the root hair plasma membrane (Goedhart et al. 1999). Moreover, in order to transduce the Nod factor signal across the plasma membrane, it is likely that putative Nod factor receptors are located within the plasma membrane. In this study, we compare the binding of nonsulfated and sulfated Nod factors on the legume species $V$. sativa and $M$. truncatula. Novel fluorescent-sulfated Nod factors are described and are used for studying root hair deformation, location, and diffusional behavior in situ on both host and nonhost legume root hairs. These studies enable us to directly assess the influence of the sulfate group on the Nod factor binding and perception in vivo.

\section{RESULTS}

\section{Fåhreus slide system for the study of M. truncatula root hairs.}

Root hair deformation on V. sativa grown in Fåhreus slides has been used as an assay for bioactivity of Nod factors (Heidstra et al. 1994). This assay is relatively fast and semiquantitative, and the observation of root hairs can be done in real time by (fluorescence) microscopy. In previous studies, this system was used to evaluate the bioactivity of fluorescent LCO-IV, its location on the root hairs, and its diffusional behavior (Gadella et al. 1997; Goedhart et al. 1999, 2000).

In order to study fluorescent sulfated LCOs and to compare the results with the nonsulfated derivatives, it was necessary to evaluate whether the Fåhreus slide system, used for $V$. sativa, was also suitable for $M$. sativa or $M$. truncatula. It appeared that, upon addition of plant growth medium, lacking Nod factors, morphogenic responses such as swelling were induced in the root hairs of $M$. sativa, rendering these plants useless for deformation studies in our Fåhreus slide system (data not shown). The root hairs of $M$. truncatula retained their normal morphology after transfer to the Fåhreus slide. After application of $10^{-9} \mathrm{M}$ NodSm-IV (Ac, S) (the major Nod factor produced by $S$. meliloti), clear root hair deformations were obtained within $3 \mathrm{~h}$, whereas replacing the medium without Nod factor did not show any effect. Hence, we decided to focus on $M$. truncatula as the host plant for studying sulfated LCOs in the deformation assay. An advantage of the M. truncatula root hairs is that the air-grown root hairs maintain their cytoarchitecture after transfer from the agarose plates to plant growth medium (PGM)-containing Fåhreus slides, which is in contrast with root hairs of $V$. sativa.

\section{Bioactivity of sulfated Nod factors.}

The Fåhreus system can be used for root hair deformation tests on $M$. truncatula; therefore, we used this assay to examine the bioactivity of the fluorescent sulfated Nod factor analogs. Three fluorescent sulfated Nod factors were used: LCOIV(BODIPY FL-C $16, S$ ), LCO-V(BODIPY FL-C $16, S$ ), and LCO-IV(BODIPY 558/568-C ${ }_{12}, \mathrm{~S}$ ) (Fig. 1). The bioactivity of these Nod factors is indicated in the root hair deformation assay on $M$. truncatula (Table 1). All three Nod factors are bioactive at concentrations down to $1 \mathrm{nM}$, although the activity of LCO-IV(BODIPY FL- $\left.\mathrm{C}_{16}, \mathrm{~S}\right)$ is reduced at this concentration.

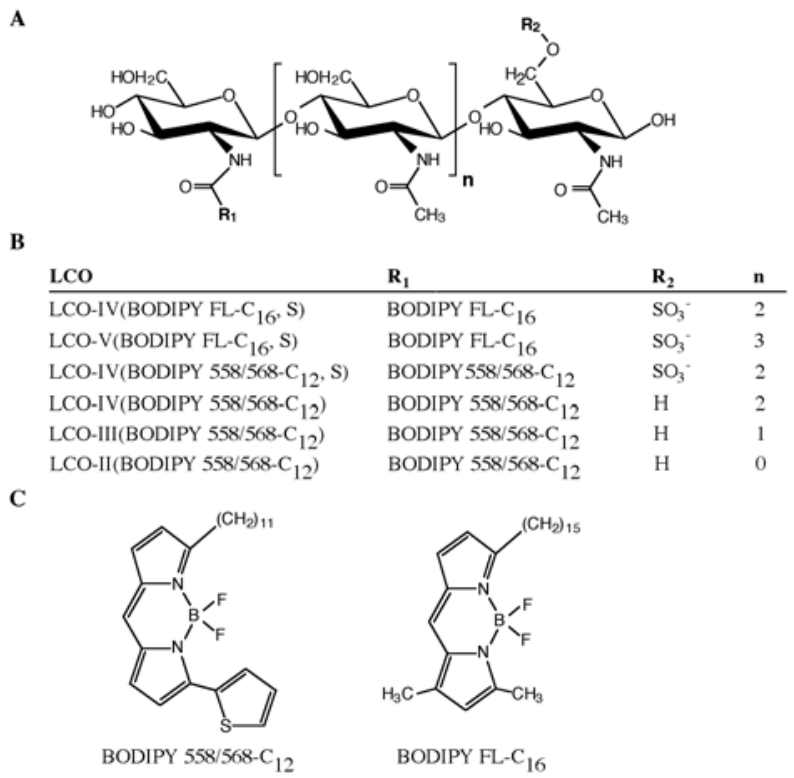

Fig. 1. Structures of the fluorescent lipo-chitooligosaccharides (LCOs) described in this study. A, Chitin-like head group with different acyl chains $\left(\mathrm{R}_{1}\right)$, sulfated or nonsulfated $\left(\mathrm{R}_{2}\right)$, and different degrees of $\mathrm{N}$ acetylglucosamine polymerization referred to as pentamers $(n=3)$, tetramers $(n=2)$, trimers $(n=1)$, and dimers $(n=0)$. B, Novel fluorescent sulfated Nod factors have two different fluorescent acyl chains, either the green fluorescent BODIPY FL- $\mathrm{C}_{16}$ or the orange fluorescent BODIPY 558/568-C 12 . C, Note that LCO-IV(BODIPY $\left.558 / 568-\mathrm{C}_{12}\right)$ is identical to the previously described NodRlv-IV (BODIPY 558/568-C 12 ) (Goedhart et al. 1999). 
The orange fluorescing Nod factor derivative, LCOIV(BODIPY 558/568- $\mathrm{C}_{12}, \mathrm{~S}$ ), induces root hair deformation even at $10^{-10} \mathrm{M}$, reflecting an order of magnitude higher bioactivity than the green fluorescing sulfated Nod factors. In contrast, none of the three sulfated fluorescent LCOs was capable of inducing root hair deformation on the nonhost legume $V$. sativa at the highest concentration tested of $10^{-7} \mathrm{M}$ (data not shown).

\section{Intermembrane transfer but no flip-flop of sulfated LCOs.}

To examine the dynamics of membrane association and intramembrane flip-flop of sulfated LCOs, we designed a transfer assay similar to that described previously for nonsulfated Nod factors (Goedhart et al. 1999). Phospholipid vesicles containing sulfated LCOs and a nontransferable fluorescence quencher were prepared by injection of an ethanolic solution of phospholipids, Texas Red DHPE, and LCOIV(BODIPY FL- $\mathrm{C}_{16}, \mathrm{~S}$ ) in aqueous buffer solution. As can be inferred from Figure 2, the fluorescence of LCOIV(BODIPY FL- $\mathrm{C}_{16}, \mathrm{~S}$ ) is very low directly after the ethanol

Table 1. Three fluorescent sulfated Nod factors were tested for their root hair deforming activities on Medicago truncatula ${ }^{\text {a }}$

\begin{tabular}{|c|c|c|c|c|}
\hline Nod factor & $10^{-8} M$ & $10^{-9} \mathrm{M}$ & $10^{-10} \mathrm{M}$ & $10^{-11} \mathrm{M}$ \\
\hline LCO-IV(BODIPY FL-C $16, \mathrm{~S}$ ) & + & \pm & - & nt \\
\hline LCO-V(BODIPY FL-C $16, S)$ & + & + & - & nt \\
\hline LCO-IV(BODIPY 558/568-C $12, \mathrm{~S})$ & + & + & + & - \\
\hline
\end{tabular}

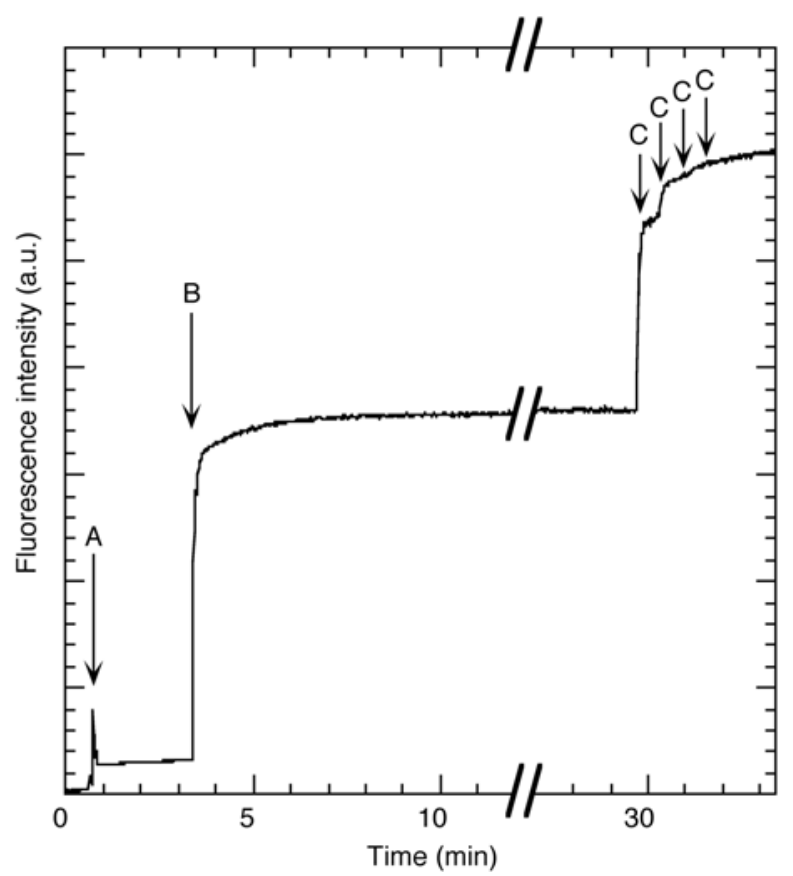

Fig. 2. Intermembrane transfer of lipo-chitooligosaccharide (LCO)IV(BODIPY FL-C $16, S$ ) from quenched donor to unquenched acceptor vesicles. The fluorescence intensity of LCO-IV(BODIPY FL-C ${ }_{16}, S$ ) is measured in arbitrary units (a.u.). At the arrow indicated with A, donor vesicles are generated by injection of an ethanolic solution (containing DOPC:DPPA:Texas Red DHPE:LCO-IV[BODIPY FL-C $\left.{ }_{16}, \quad \mathrm{~S}\right]=$ 79:10:10:1 mol\%, $20 \mathrm{nmol}$ total lipid) into $3 \mathrm{ml}$ of Tris buffer under continuous stirring. For initiating spontaneous transfer, a 10-fold excess of acceptor vesicles (containing DOPC:DPPA $=90: 10 \mathrm{~mol} \%$ ) was added at the arrow indicated with B. After four subsequent additions of $25 \mu \mathrm{l}$ of $10 \%$ (vol/vol) Triton X-100 in phosphate-buffered saline (indicated by arrow $\mathrm{C}$ ), the vesicles are completely solubilized, thereby dispersing and dequenching LCO-IV(BODIPY FL-C $\left.{ }_{16}, \mathrm{~S}\right)$. injection (see arrow A). The very low fluorescence is caused by quenching by the fluorescence resonance energy transfer (FRET) acceptor Texas Red DHPE, which is present in the vesicles. Because FRET occurs only at distances less than 10 $\mathrm{nm}$, this result demonstrates quantitative association of the Nod factor with the vesicles. The subsequent addition of a 10 -fold excess of unlabeled acceptor vesicles results in a fast increase in fluorescence, indicating that the Nod factors are rapidly transferred from the quenched donor vesicles to the unquenched acceptor vesicles. The increased level of fluorescence intensity remained stable over at least $25 \mathrm{~min}$. Only after the addition of Triton X-100, which disrupts all vesicles, thereby releasing the remaining quenched Nod factors, did the fluorescence intensity increase. These results show that approximately $40 \%$ of the fluorescent Nod factors are not available for transfer to the acceptor vesicles and, therefore, remain within the quenched vesicles. These observations can be explained by the fact that Nod factors located in the inner leaflet of the original donor vesicles will have to flip to the outer leaflet of the vesicle before transfer can occur. Hence, within the $25 \mathrm{~min}$ of observation time, no transmembrane flip-flop of sulfated Nod factors takes place. These results are very similar to the results obtained for nonsulfated Nod factor (Goedhart et al. 1999).

\section{Chitinase activity on roots.}

Plant roots exhibit Nod factor hydrolyzing activity (Heidstra et al. 1994; Staehelin et al. 1994), which prompted us to study to what extent Nod factor breakdown products accumulate when the fluorescent Nod factors are applied to plants. Therefore, we extracted and analyzed the fluorescent components from the roots and medium after different incubation times. $V$. sativa root systems were incubated with $500 \mathrm{nM}$ LCO-IV(BODIPY 558/568- $\mathrm{C}_{12}$ ) for 10, 30, 60, and $750 \mathrm{~min}$, after which both the medium was isolated and the roots were ground. Nod factors were isolated from both fractions by n-

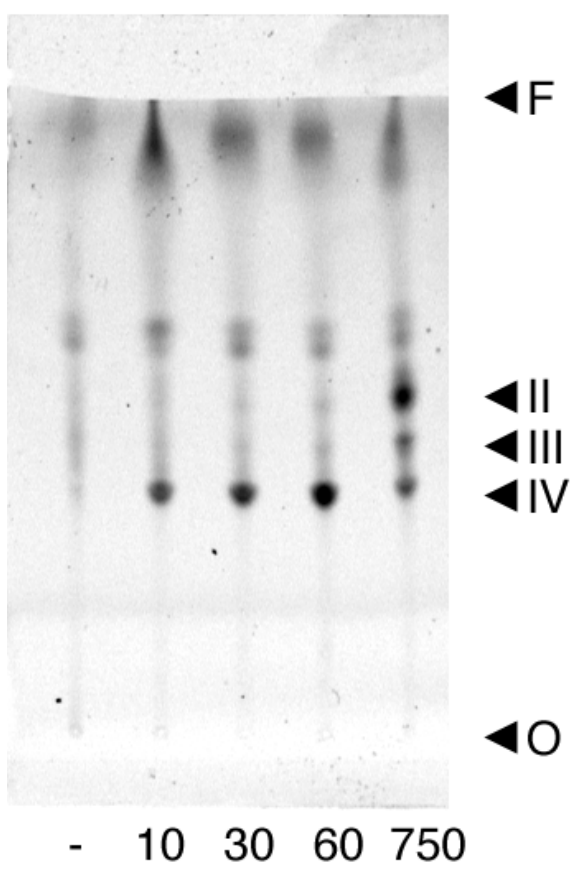

Fig. 3. Thin-layer chromatography analysis of Nod factor hydrolyzing activity of Vicia sativa roots. Root extracts before and 10, 30, 60, or 750 min after addition of $500 \mathrm{nM}$ lipo-chitooligosaccharide (LCO)IV(BODIPY 558/568- $\mathrm{C}_{12}$ ), showing the degradation to LCO-III(BODIPY $558 / 568-\mathrm{C}_{12}$ ) and LCO-II(BODIPY 558/568- $\mathrm{C}_{12}$ ). O and $\mathrm{F}$ denote the location of sample application and solvent front, respectively. 
butanol extraction. When the extracts from unlabeled roots were analyzed by thin-layer chromatography (TLC), it was observed that some fluorescent compounds from the root were present in the butanol layer, as can be seen in the first lane of the TLC in Figure 3. Still, the Nod factor fluorescence can be detected clearly above the background fluorescence. From the TLC analysis of the root extracts (Fig. 3), it can be inferred that no breakdown of Nod factors is detected within the first $10 \mathrm{~min}$. After $30 \mathrm{~min}$, less than $5 \%$ of the Nod factors was degraded; however, after prolonged incubation, the majority of the Nod factors was degraded to LCOIII(BODIPY 558/568-C ${ }_{12}$ ) and LCO-II(BODIPY 558/568$\mathrm{C}_{12}$ ). Extracts of the medium after 10 to $60 \mathrm{~min}$ showed only LCO-IV(BODIPY 558/568-C 12 ), whereas LCO-II(BODIPY $\left.558 / 568-\mathrm{C}_{12}\right)$ was the major compound $(>50 \%)$ present after 750 min (data not shown). The kinetics of Nod factor hydrolysis by roots corresponds with previous studies using radiolabeled compounds (Heidstra et al. 1994). Essentially similar results were obtained for either LCO-IV(BODIPY $\left.558 / 568-\mathrm{C}_{12}, \mathrm{~S}\right)$ or the nonsulfated derivative upon application to $M$. truncatula roots (data not shown).
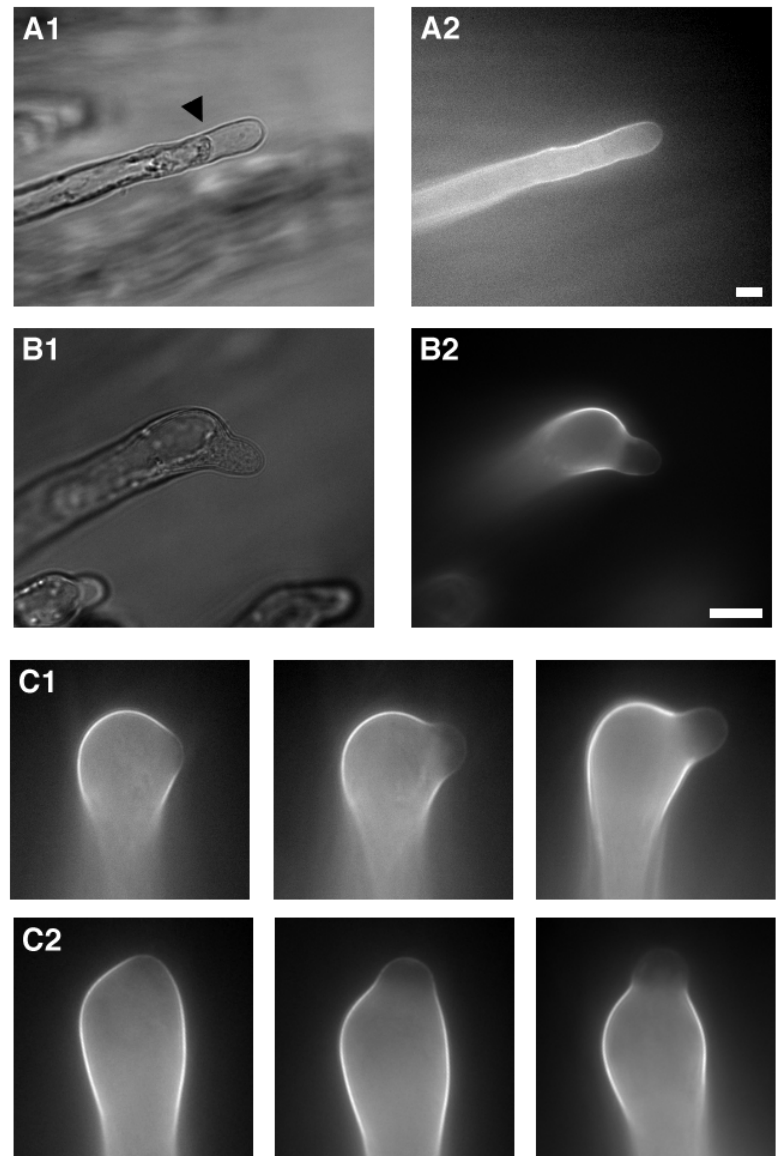

Fig. 4. Digital images of Medicago truncatula incubated with $10 \mathrm{nM}$ lipo-chitooligosaccharide (LCO)-IV(BODIPY 558/568-C ${ }_{12}, \mathrm{~S}$ ). After 10 min, the PGM is replaced with $0.45 \mathrm{M}$ mannitol to induce plasmolysis A1, which can be seen in the phase contrast images as a retraction of the plasma membrane from the cell wall. The plasma membrane is indicated with an arrow head. A2, Fluorescence images show the predominant location of the fluorescent Nod factor in the cell wall. After $3 \mathrm{~h}$, the root hairs are deformed due to the presence of the Nod factor. B1, New outgrowth is clearly visible in the phase contrast images. B2, Fluorescence image represents the location of LCO-IV(BODIPY 558/568- $\mathrm{C}_{12}, \mathrm{~S}$ ) showing a lack of fluorescence in the new outgrowth. C1 and C2, Highmagnification images of root hairs at different stages of root hair deformation. Bars represent $15 \mu \mathrm{m}$, and the full width of the individual images of $\mathrm{c} 1$ and $\mathrm{c} 2$ is $68 \mu \mathrm{m}$.
These results demonstrate that Nod factor breakdown products are a minor fraction and, thereby, suggest that they do not interfere with in vivo measurements which are performed within $60 \mathrm{~min}$ after application of Nod factors. Furthermore, the prolonged incubations show that the presence of the BODIPY moiety on the acyl chain does not perturb chitinase activity. The time course of chitinase activity suggests no involvement of breakdown products in early signaling responses, but does not exclude their involvement in processes at a later stage (e.g., infection thread initiation).

\section{Location of fluorescent LCOs}

on $V$. sativa and $M$. truncatula roots.

Although the bioactivity of the green fluorescent Nod factor derivatives is relatively high, they are less well suited for in vivo localization studies by fluorescence microscopy at physiological relevant concentrations due to a relative high contribution of root autofluorescence at these excitation or emission wavelengths (Gadella et al. 1997). However, red-shifted fluorescent Nod factor derivatives have excellent spectral properties for localization and diffusional studies at a relatively low concentration, as shown before (Goedhart et al. 1999, 2000). The spectroscopic properties of the novel LCO-IV(BODIPY $\left.558 / 568-\mathrm{C}_{12}, \mathrm{~S}\right)$ are identical to those described for the nonsulfated Nod factor (Goedhart et al. 1999), and ideally suited for in vivo labeling studies.

In spite of the advantageous spectral properties, it is not possible to image the orange fluorescent LCO at the threshold concentration of biological activity of $0.1 \mathrm{nM}$ (data not shown). To obtain images with a reasonable signal-to-background level, the LCOs need to be applied at a concentration of $10 \mathrm{nM}$ or higher. Upon application of $10 \mathrm{nM}$ LCOIV(BODIPY 558/568- $\mathrm{C}_{12}, \mathrm{~S}$ ) to the roots of M. truncatula, an increase in fluorescence on the root hairs was detected, with a concomitant decrease of fluorescence in the medium near the root hair tips. Fluorescence imaging revealed surface labeling of the root hairs. To verify whether the fluorescence repre-

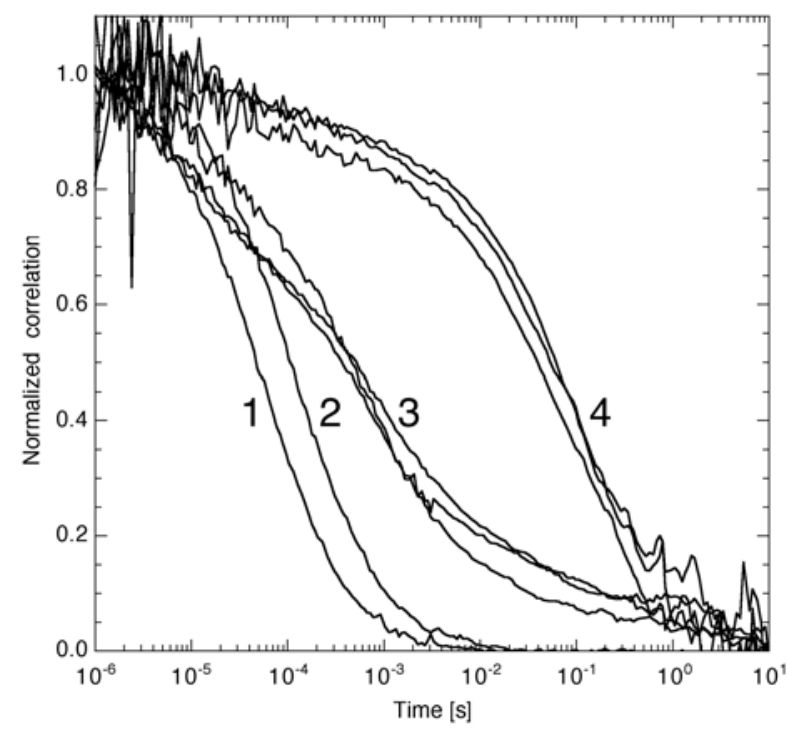

Fig. 5. Normalized autocorrelation curves of carboxy-tetramethylrhodamine (TMR) and lipo-chitooligosaccharide (LCO)-IV(BODIPY 558/568$\mathrm{C}_{12}, \mathrm{~S}$ ) measured by fluorescence correlation microscopy. The confocal volume element was positioned in the medium $(1,2)$ to measure the diffusion of TMR (1) or LCO-IV(BODIPY 558/568-C $12, \mathrm{~S})(2)$ or at the tip of Medicago truncatula susceptible (zone II) root hairs to measure the mobility of TMR (3) or LCO-IV(BODIPY 558/568- $\mathrm{C}_{12}, \mathrm{~S}$ ) (4). For the measurement in root hairs $(3,4)$ representative curves from three individual root hairs are shown. 
sented cell wall or plasma membrane labeling, plasmolysis was induced by replacing the medium with a $0.45 \mathrm{M}$ mannitol solution. After the plasma membrane was withdrawn from the cell wall, all the fluorescence still was associated with the cell wall and no fluorescence was observed in the plasma membrane (Fig. 4A). It is of note that the acyl chain (BODIPY 558/568$\mathrm{C}_{12}$ ) alone accumulates immediately in the cytoplasm of $M$. truncatula root hairs (data not shown), as previously observed for $V$. sativa (Goedhart et al. 2000).

When the seedlings were incubated for $3 \mathrm{~h}$ with $10 \mathrm{nM}$ LCO-IV(BODIPY 558/568- $\mathrm{C}_{12}, \mathrm{~S}$ ), the root hairs were clearly deformed. As can be inferred from Figure 4B, the newly formed outgrowths were hardly labeled, whereas the older part of the root hair was still clearly fluorescent, indicating a lack of diffusion of the LCO through the cell wall. A high resolution series of fluorescent root hairs during different stages of root hair deformation (Fig. 4C) shows that initiation of the new outgrowth is paralleled by a local decrease in cell wall labeling, indicating the insertion of new unlabeled cell wall material at this position.

\section{FCM of fluorescent LCOs.}

The plasmolysis and deformation experiments point to affinity of Nod factors for the cell wall and a reduced mobility of the sulfated Nod factors in the root hair cell wall as observed for $V$. sativa labeled with nonsulfated Nod factors. In order to obtain more detailed information on the behavior of the fluorescent LCO on root hairs, FCM experiments were performed. A focused laser beam was positioned at the preferred location in the specimen and the fluorescence intensity fluctuations originating from the confocal volume element were measured. These fluctuations were autocorrelated, yielding information on the diffusion time that is necessary for the fluorescent molecules to cross the volume element. Additionally, the amplitude of the autocorrelation is inversely related to the average number of fluorescent molecules inside the volume element. By fitting the autocorrelation curve, both the diffusion time and the particle number can be determined. When the dimensions of the confocal volume element are determined by calibration, the diffusion constant (see equation 1) and the concentration of the fluorescent molecules can be calculated (Goedhart et al. 2000).

Autocorrelation curves obtained from $10 \mathrm{nM}$ LCOIV(BODIPY 558/568- $\mathrm{C}_{12}, \mathrm{~S}$ ) in PGM represented diffusion of monomers (Fig. 5, curve 2), as observed before, for nonsulfated fluorescent LCOs in aqueous media (Goedhart et al. 1999, 2000). Autocorrelation curves obtained from intensity fluctuations measured in root hair tips after addition of $10 \mathrm{nM}$ LCO-IV(BODIPY 558/568- $\mathrm{C}_{12}, \mathrm{~S}$ ) to the roots of $M$. truncatula are reported in Figure 5 (three representative curves indicated with "4"). Qualitatively, these curves represent very long diffusion times, typical for molecular interactions on immobilized structures (Brock et al. 1999; Schwille et al. 1999). The greatly reduced mobility of sulfated Nod factor on $M$. truncatula (three orders of magnitude) compares favorably with the immobility of their nonsulfated counterparts on $V$. sativa (Goedhart et al. 2000).

To further examine whether this reduced mobility results from nonspecific interactions, we measured the mobility of carboxy-tetramethylrhodamine (TMR) in the presence of root hairs as well. TMR is a standard fluorescent compound often used to calibrate FCM measurements because it has a known diffusion constant and optimal spectroscopic properties for 543-nm excitation, yet it has no known biological function. Interestingly, upon application of TMR to Medicago spp. roots, a moderate accumulation in the root hair cell wall but no internalization into the root hair cytosol was observed, qualitatively mimicking Nod factors. The diffusional behavior of TMR in plant growth medium was identical to the reference situation in pure $\mathrm{H}_{2} \mathrm{O}$ (Fig. 5, curve 1). When the confocal volume element was positioned in the cell wall at the tip of a growing root hair and TMR-fluorescence intensity fluctuations were monitored, autocorrelation curves were shifted to longer times (Fig. 5, three representative curves indicated with " 3 "). This indicates that the mobility of TMR is reduced in root hair cell walls relative to diffusion in aqueous solution. However, TMR was still two orders of magnitude more mobile than Nod factors in root hair cell walls. Furthermore, for TMR, it was possible to acquire proper autocorrelation curves in the older root hair cell wall further from the tip, whereas this was not possible for fluorescent LCOs due to very strong immobilization and, consequently, severe bleaching (data not shown). These data demonstrate that Nod factor binding to cell walls is strong, and clearly surpasses levels that can be explained by nonspecific interactions such as those observed with TMR.

Using FCM, we analyzed the mobility of the sulfated and nonsulfated Nod factors on the surface of root hairs of either $M$. truncatula or $V$. sativa. To enable a good comparison, all measurements were performed by positioning the confocal volume in the cell wall at the tip of nearly full-grown root hairs. When the autocorrelation curves of TMR and fluorescent LCOs in root hair cell walls were analyzed, two diffusion constants were necessary for a proper fit, as described before (Goedhart et al. 2000). The obtained parameters are listed in Table 2. Interestingly, the diffusion constants for TMR and LCOs were quite similar. However, the fast diffusion constant was most significant for TMR (contributing at least $75 \%$ to the curve), whereas the contribution of the fast diffusion constant was small for fluorescent Nod factors. For all Nod factors, the slower diffusion constant contributed 80 to $90 \%$ and had an average value of $2.1 \times 10^{-13}$ to $3.3 \times 10^{-13} \mathrm{~m}^{2} / \mathrm{s}$. This is 1,000 -fold

Table 2. Results of the analysis of the autocorrelation curves obtained by fluorescence correlation microscopy of root hair cell walls

\begin{tabular}{lllclrrr}
\hline Plant & Probe $^{\mathbf{a}}$ & $\boldsymbol{n}^{\mathbf{b}}$ & $\mathbf{D 1}\left(\times \mathbf{1 0}^{\mathbf{1 1}}\right) \mathbf{~ m}^{\mathbf{2} / \mathbf{s}^{\mathbf{c}}}$ & $\mathbf{\%} \mathbf{D 1}$ & $\mathbf{D 2}\left(\times \mathbf{1 0}^{\mathbf{1 3}}\right) \mathbf{m}^{\mathbf{2} / \mathbf{s}^{\mathbf{d}}}$ & $\mathbf{N}^{\mathbf{e}}$ & $\mathbf{c}(\mathbf{n M})^{\mathbf{f}}$ \\
\hline Medicago truncatula & TMR & 8 & $2.3 \pm 0.5$ & $82 \pm 6$ & $2.3 \pm 1.3$ & $25 \pm 6$ & $53 \pm 13$ \\
M. truncatula & LCO-IV(S) & 7 & $3.2 \pm 2.3$ & $20 \pm 5$ & $2.1 \pm 0.7$ & $52 \pm 24$ & $113 \pm 52$ \\
M. truncatula & LCO-IV & 8 & $4.8 \pm 3.7$ & $22 \pm 5$ & $2.3 \pm 1.4$ & $44 \pm 16$ & $90 \pm 33$ \\
Vicia sativa & LCO-IV(S) & 8 & $4.7 \pm 2.3$ & $16 \pm 9$ & $3.2 \pm 0.9$ & $72 \pm 36$ & $147 \pm 74$ \\
V. sativa & LCO-IV & 7 & $8.2 \pm 7.3$ & $13 \pm 2$ & $3.3 \pm 1.0$ & $57 \pm 9$ & $112 \pm 18$ \\
\hline
\end{tabular}

${ }^{a}$ Fluorescent Nod factor derivatives carry the BODIPY 558/568- $\mathrm{C}_{12}$ acyl chain; TMR = carboxy-tetramethylrhodamine and LCO $=$ lipochitooligosaccharide.

${ }^{\mathrm{b}}$ Number of experiments.

${ }^{c}$ Average fast diffusion constant ( \pm standard deviation $\left.[\mathrm{SD}]\right)$.

$\mathrm{d}$ Average slow diffusion constant $( \pm \mathrm{SD})$.

e Average number of molecules in the confocal volume $( \pm \mathrm{SD})$.

${ }^{\mathrm{f}}$ Average concentration in the confocal volume $( \pm \mathrm{SD})$ calculated from $\mathrm{N}$ and the volume determined by calibration. Considering that the cell wall occupies approximately $25 \%$ of the volume element (Goedhart et al. 2000), the Nod factor concentration in the cell wall is approximately fourfold higher than the tabulated values. 
reduced compared with the value of $2.68 \times 10^{-10} \mathrm{~m}^{2} / \mathrm{s}$ for free Nod factor (Goedhart et al. 1999). Remarkably, no difference was observed when the sulfated and nonsulfated LCOs were compared; in addition, the diffusional mobility on $V$. sativa and $M$. truncatula was similar. These results indicate that the accumulation and mobility of Nod factors does not depend on the presence of the sulfate group, and is comparable in $V$. sativa and M. truncatula.

\section{Depletion of LCOs from the medium.}

The observation that the new outgrowth after deformation is unstained with fluorescent LCO implies that the root hair cell walls very efficiently absorb Nod factors from the medium. By positioning the confocal volume element of the FCM in the growth medium at different distances from the root hairs, it was observed that the Nod factor concentration in the medium near the root hairs was lower than the Nod factor concentration further away from the root (data not shown), suggesting a localized depletion.

To visualize the depletion of fluorescent Nod factors from the medium, the root system of $M$. truncatula was incubated with $10 \mathrm{nM}$ LCO-IV(BODIPY 558/568- $\mathrm{C}_{12}$, S) and digital images of the fluorescence in the medium surrounding the root were captured using a stereoscope equipped with a highly sensitive liquid-cooled CCD-camera. Because of the low numerical aperture of the stereoscope lens and the very low amount of label used, long exposure and camera integration times were needed. A series of fluorescence images of the root and the surrounding medium is shown in Figure 6 . The first image already shows that the medium close to the root hairs is less fluorescent than the medium further away from the root. During time, the fluorescence intensity of the medium decreases, with the most dramatic decrease near the root hairs. In this way, the root creates a concentration gradient of fluorescent LCOs in the growth medium. It is remarkable that the strongest depletion is observed near the region of the root containing young root hairs, which are predominantly susceptible to infection by rhizobia. These results demonstrate the remarkable capability of root hairs to absorb and concentrate Nod factors and for the first time directly and quantitatively visualize the depletion of Nod factors from the medium. Interestingly, when the radiolabeled Nod factor (NodSm-IV[Ac, 35S, C16:2]) was applied for $3 \mathrm{~h}$ to the root system, almost all of the initially added radioactivity could be recovered from the medium (data not shown). These results agree with earlier experiments by Heidstra and associates (1996) showing that 1 to $2 \%$ of the applied Nod factors can be bound to the roots. Together, these results show that the Nod factor depletion zone does not extend far into the bulk medium.

\section{DISCUSSION}

In this article, we describe the results that were obtained using fluorescent Nod factors to probe Nod factor perception. Using the root hair deformation assay as a measure of bioactivity of the fluorescent LCOs, it was shown that the novel BODIPY 558/568- $\mathrm{C}_{12}$-labeled sulfated LCO is remarkably active, with a threshold concentration of $10^{-10} \mathrm{M}$ on $\mathrm{M}$. truncatula root hairs. This is equal to the highest bioactivity of a nonsulfated fluorescent Nod factor analog measured on V. sativa in a similar assay (Goedhart et al. 1999). It is interesting to note that, for $V$. sativa, nonsulfated LCOs with a BODIPY FL-C ${ }_{16}$ acyl chain are more active than those labeled with BODIPY 558/568-C $\mathrm{C}_{12}$ (Goedhart et al. 1999); whereas, for M. truncatula, the opposite is observed with the corresponding sulfated derivatives. This difference in acyl chain preference between the two species points to specific recognition of the acyl chain and might reflect the fact that the putative Nod factor signaling receptors in the two species have a structurally different lipidbinding pocket.

A FRET-based transfer assay showed that LCO-IV(BODIPY FL- $\left.\mathrm{C}_{16}, \mathrm{~S}\right)$ cannot flip-flop through a lipid bilayer. Consequently, as postulated for nonsulfated Nod factors (Goedhart et al. 1999), an active flip-flop mechanism is required for secretion of sulfated Nod factors by rhizobia. For LCO-IV(BODIPY $\left.558 / 568-\mathrm{C}_{12}, \mathrm{~S}\right)$, it is clear from FCM experiments that it diffuses as monomers in plant growth medium when applied at a concentration of $10 \mathrm{nM}$. Hence, the insertion of LCOs into membranes and their ability to diffuse as monomers at low concentrations in aqueous solution is hardly or not dependent on the presence of the sulfate group.

When $M$. truncatula roots were incubated with growth medium containing LCO-IV(BODIPY 558/568- $\mathrm{C}_{12}$, S), the fluorescent LCO accumulated in the cell walls of $M$. truncatula root hairs. The plasmolysis experiments show the absence of fluorescence associated in plasma membrane, when these LCOs are applied at $10 \mathrm{nM}$. The strong binding to the cell wall also was evident from the lack of diffusion of Nod factor to the newly formed outgrowth induced by the Nod factors. It is of interest that the initial binding of Nod factors to root hair cell walls of a compatible and noncompatible host (sulfated LCOs on $V$. sativa and nonsulfated LCOs on $M$. truncatula) is similar.

To study the binding characteristics at the molecular level, the diffusional behavior and accumulation of Nod factors was measured by FCM. Quantitative analysis of the FCM autocorrelation curves showed marked immobilization but no difference in diffusional characteristics and accumulation of sulfated and nonsulfated LCOs on root hair cell walls of compatible and noncompatible legumes. Although binding of the Nod factor to the cell wall needs the chitin backbone, because the free acyl chain is directly taken up into the root hair cytosol, the presence of a sulfate group changes neither the extent nor the location of binding.

For comparison, a biologically nonrelevant fluorophore, TMR, was included in the FCM analysis. TMR showed moderate accumulation in root hair cell walls and a slower diffusion compared with diffusion in aqueous medium. However, the average diffusion of TMR in root hair cell walls is still 100fold faster than the diffusion of fluorescent LCOs. This clearly shows that Nod factors bind more tightly to the cell wall than nonspecific lipophilic molecules such as TMR.

For our analysis of the FCM data, we used a two component model. This could suggest that Nod factors move as two rather independent populations of faster and slower diffusing molecules in a root hair cell wall. However, the theory underlying the twocomponent model assumes a homogeneous solution of fluores-

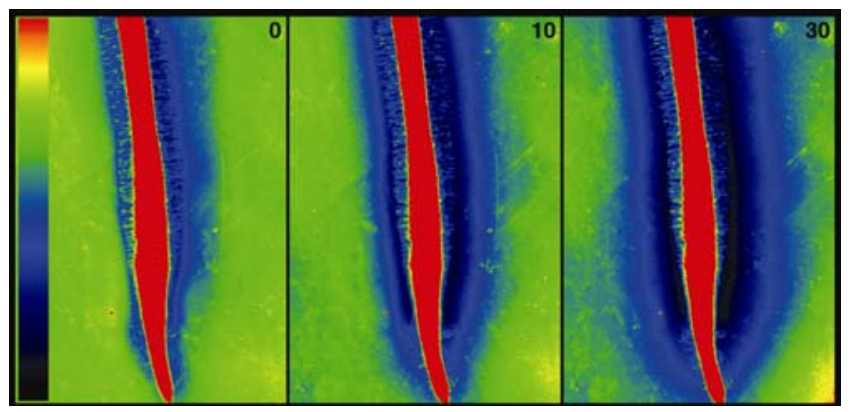

Fig. 6. Fluorescent Nod factors are depleted from the growth medium by the Medicago truncatula root. Fluorescent lipo-chitooligosaccharide (LCO)-IV(BODIPY 558/568-C 12 , S) $(10 \mathrm{nM})$ was added to the medium of a $M$. truncatula root, after which the fluorescence was imaged. The time series (time in minutes) shows the decrease of fluorescence intensity in the medium, especially in areas close to the root. 
cent particles continuously occupying the confocal volume element (Maiti et al. 1997). It is highly likely that this assumption does not apply to our system, because (i) the cell wall (thickness approximately $150 \mathrm{~nm}$ ) (Goedhart et al. 2000) is too thin to occupy the volume element completely, (ii) we do not know whether the diffusion in all parts of the cell wall is similar (e.g., if diffusion parallel and perpendicular to the root hair axis is the same), and (iii) the cell wall contains aqueous microdomains separated by cell wall polymers yielding hindered diffusion due to volume exclusion. In an attempt to account for volume exclusion effects, we tried to fit the data to an anomalous diffusion model (Schwille et al. 1999). The anomalous fits were worse than the two component fits (data not shown), suggesting that volume exclusion does not solely account for the observed diffusion behavior. Considering the problems of attributing the twocomponent model, we would rather refer to the data as "complex diffusion," also observed by others in different cellular systems (Brock et al. 1998, 1999; Schwille et al. 1999). Despite the above factors, the curves observed for LCOs and TMR on root hairs differ markedly from each other and from the curves obtained in solution (Fig. 5; note the logarithmic scale of the horizontal axis). These qualitative differences clearly show the usefulness of the FCM technique in comparing diffusion behavior of molecules in living cells.

Based on our results, we propose that sulfate-independent Nod factor binding and immobilization is accomplished by a Nod factor binding factor (NFBF) located in the cell wall. Interestingly, a previously reported biochemical analysis of a Nod factor binding site in Medicago spp. root extracts (NFBS1) indicated that (i) this site was most abundant in a high-density fraction and not in a membrane fraction (Bono et al. 1995), (ii) a similar binding site was identified in tomato root extracts, (iii) both the chitin and acyl chain moieties were required for binding to NFBS1, but (iv) the absence or presence of a sulfate group did not alter the binding affinity (Bono et al. 1995). These data fit very well with our observations because (i) we see Nod factor binding in situ in the cell wall and not in the plasma membrane; (ii) in addition, root hairs of nonleguminous plants exhibit a certain (albeit reduced) Nod factor binding capacity (Goedhart et al. 2000); (iii) removal of the chitin backbone of the Nod factor yields cytoplasmic labeling; and (iv) we see comparable binding and immobilization of sulfated and nonsulfated Nod factors in situ. Hence, it is likely that NFBS1 is the Nod factor binding factor that we identified in the root hair cell wall in our in situ studies.

Because of the high Nod factor-binding capacity of the cell wall, this binding factor apparently is relatively abundant. Its biological function could be to prevent lateral diffusion of Nod factors in the cell wall after secretion by Rhizobium bacteria, as proposed previously (Goedhart et al. 2000). In this way, the highest concentration of Nod factors in the cell wall is reached and maintained at the site where the rhizobia reside (Fig. 7). This accomplishes a pinpointing mechanism, possibly spatially controlling the root hair curling process and infection thread initiation point. If, in contrast, Nod factors could diffuse laterally and through the cell wall at a high speed, a delocalized Nod factor signal would appear in the cell wall, with no clear spatial correlation between the site of Nod-factor secretion (by rhizobia) and the site of Nod-factor perception.

It is clear that besides the binding in the cell wall as described above, another step is involved in the actual triggering of the signal transduction cascade leading to root hair deformation. This factor, the putative signaling receptor, should possess recognition sites and, hence, different affinities for special features on Nod factors, including the presence or absence of the sulfate group and variations in the acyl chain. We cannot meas- ure differences in affinity for Nod factors without or with sulfate groups or Nod factors with different acyl chains; this implies that NFBF is at least two orders of magnitude more abundant than the putative signaling receptor. The recently identified NORK could be a candidate for this signaling receptor (Endre et al. 2002; Stracke et al. 2002). NORK is predicted to be membrane bound and contains an extracellular leucinerich repeat (LRR) which fits a multicomponent perception system, as also proposed by others (Endre et al. 2002; Stracke et al. 2002; Van der Holst et al. 2001).

If we assume two components being involved in Nod factor perception (NFBF and NORK), two models can be discriminated, which are schematically depicted in Figure 7: model A, in which both act independently, and model B, in which the two components interact physically. Model A predicts that the NFBF is involved only in Nod factor immobilization and that the actual recognition of Nod factors is performed by the signaling receptor. Therefore, in this model, the receptor should be rather immobile in the plasma membrane (e.g., by linkage to the cytoskeleton) in order to maintain positional information. The receptor will have a fatty acid binding pocket in order to recognize special features on the lipid tail of the Nod factors and a binding pocket for recognition of the chitin backbone and its decorations (e.g., sulfate groups).

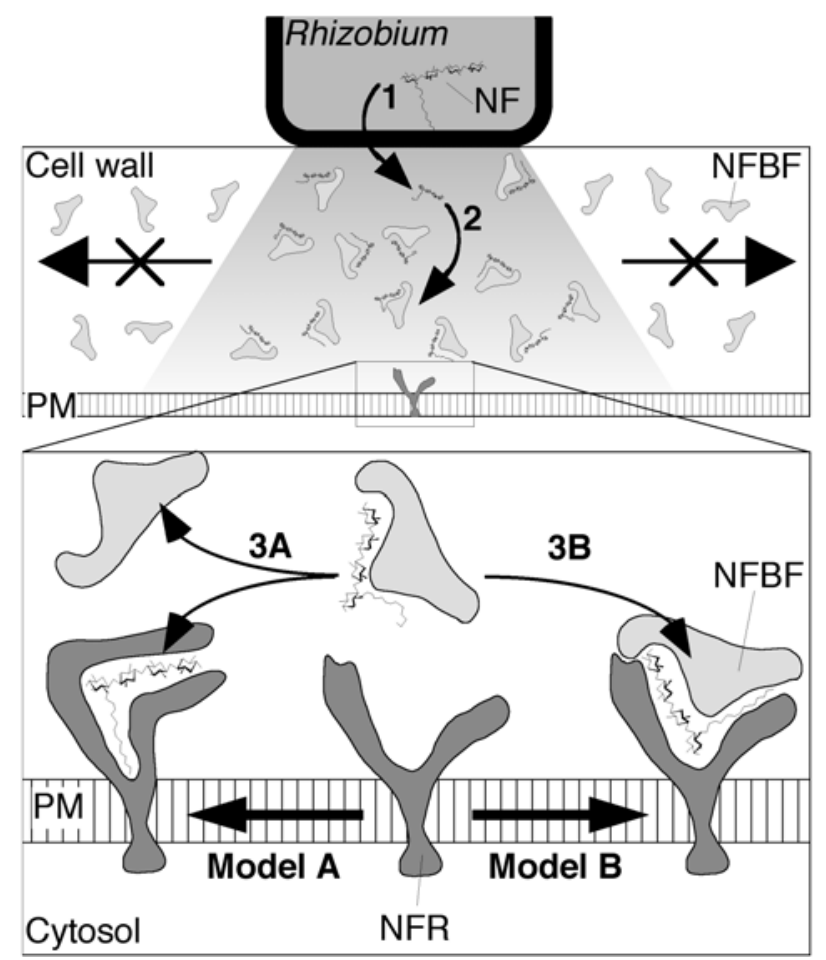

Fig. 7. Model for Nod factor perception in root hair cell walls. After secretion of Nod factors (NF) by Rhizobium spp. (arrow 1), the Nod factors bind to Nod factor binding factors (NFBFs) which are immobilized in the cell wall (arrow 2), effectively restricting lateral diffusion of Nod factors (indicated by the shadowed gradient and crossed arrows). For the transduction of the Nod factor signal to the cytosol, two mechanisms are proposed, both including the presence of a low-abundance plasma membrane (PM) spanning Nod factor signaling receptor (NFR). Model A proposes a specific Nod factor binding pocket in the NFR which will be occupied after spontaneous transfer of a Nod factor monomer from NFBF to the NFR (arrow 3A). Alternatively, in model B, a hybrid binding site is formed by NFR and NFBF in which one Nod factor is sandwiched (arrow 3B). In the latter scenario, formation of a ternary complex is needed to activate the intracellular signaling cascade; specific NF decorations still are recognized by NFR and the NFR will be immobilized in the plasma membrane after binding to the occupied NFBF. Scenario B could imply that NFR alone has no affinity for Nod factor. 
Model B predicts that NFBF both immobilizes the Nod factor and presents the Nod factor to the signaling receptor which, together with NFBF, forms a hybrid binding site for Nod factors. Only the complex of the signaling receptor and the NFBF will recognize specific features of the Nod factor: acyl chain unsaturation and length and presence of sulfate groups. Within this model, the signaling receptor could be mobile in the membrane: it would be triggered and immobilized only at the site where cell wall-bound NFBF is occupied with Nod factors. The most important consequence of model $\mathrm{B}$ is that it predicts that the signaling receptor alone might not have any affinity for Nod factors at all and that it requires the presence of NFBF. This model has striking similarity to the $\mathrm{Cf} 9$ receptor-like protein which is required to confer resistance against Cladosporium fulvum, whereas $\mathrm{Cf} 9$ displays no apparent binding affinity for the ligand (AVR9) and AVR9 binding also occurs in susceptible genotypes (de Wit et al. 1997). The LRRs on the NORK favor the possibility of protein-protein interactions necessary for perception (Endre et al. 2002; Stracke et al. 2002). This interacting protein could be the NFBF (as postulated in model B) or yet another component, yielding a more complicated version of model A (data not shown).

In summary, we have found that the major Nod factor binding activity in root hairs is independent of the sulfate group and mainly located in the cell wall. Based on these results, we have presented a model for Nod factor binding. Obviously, the combined effort of biochemical, genetic, and in situ approaches will be essential for discriminating between models A and B or yet other unforeseen models for Nod factor binding and perception in legume root hairs.

\section{MATERIALS AND METHODS}

\section{Materials.}

Synthesis of the fluorescent sulfated Nod factors will be described elsewhere (P. De Medina, S. Bedel, P. Tisnes, and C. Picard, unpublished data). The fluorescent Nod factor derivatives are named LCO-IV(BODIPY FL-C $16, \mathrm{~S})$, LCOV(BODIPY FL-C $16, S$ ), and LCO-IV(BODIPY 558/568-C ${ }_{12}$, $\mathrm{S})$. The nonsulfated Nod factor has been described previously (Goedhart et al. 1999) as NodRlv-IV (BODIPY 558/568- $\mathrm{C}_{12}$ ) and is denoted here as LCO-IV(BODIPY 558/568- $\mathrm{C}_{12}$ ). The structures of fluorescent Nod factors used in this study are shown in Figure 1.

\section{Transfer assay.}

For transfer experiments, quenched donor vesicles were prepared by injecting $20 \mu \mathrm{l}$ of an ethanolic solution of $790 \mu \mathrm{M}$ DOPC, $100 \mu \mathrm{M}$ DPPA, $10 \mu \mathrm{M}$ LCO-IV(BODIPY FL-C $16, \mathrm{~S}$ ), and $100 \mu \mathrm{M}$ Texas Red DHPE (Molecular Probes, Leiden, The Netherlands) into $3 \mathrm{ml}$ of Tris buffer (20 mM Tris, pH 7.4, 100 $\mathrm{mM} \mathrm{NaCl}, 1 \mathrm{mM}$ EDTA, and $1 \mathrm{mM}$ EGTA), under continuous stirring (Batzri and Korn 1973). Acceptor vesicles were prepared by mixing 3,600 nmol of DOPC (1,2-dioleoyl-sn-glycero-3-phosphocholine) and $400 \mathrm{nmol}$ of DPPA (1,2-dipalmitoyl;-sn-glycero-3-phosphate) in chloroform. After evaporation of the chloroform, the lipids were dissolved in $2 \mathrm{ml}$ of Tris buffer by vortexing, followed by sonication for $3 \mathrm{~min}$. The emission of NodRlv-IV(BODIPY FL-C 16 ) was monitored continuously on an Aminco SLM-8000 (SLM instruments, Urbana, IL) by excitation at $490 \mathrm{~nm}$ (slit $4 \mathrm{~nm}$ ) and emission at 513 or $550 \mathrm{~nm}$ (slit $4 \mathrm{~nm}$ ). To start the transfer, $100 \mu \mathrm{l}$ of acceptor vesicles was added to $3 \mathrm{ml}$ of donor vesicles, and the fluorescence intensity was monitored as a function of time. For calibration, an aliquot of $25 \mu \mathrm{l}$ of $10 \%$ (vol/vol) Triton X-100 in phosphate-buffered saline was added, until the fluorescence intensity did not increase.

\section{Plant material.}

Experiments on $V$. sativa were performed as described (Heidstra et al. 1994). M. truncatula ecotype A17 or alfalfa seed were treated for 5 to 10 min with concentrated $\mathrm{H}_{2} \mathrm{SO}_{4}$, washed with $\mathrm{H}_{2} \mathrm{O}$, treated with concentrated bleach for 5 to $10 \mathrm{~min}$ and washed extensively with $\mathrm{H}_{2} \mathrm{O}$. Subsequently, seeds were transferred to $1 \%$ agarose plates and incubated at $4^{\circ} \mathrm{C}$ for 2 to 3 days. After 2 days at $20^{\circ} \mathrm{C}$, seedlings were transferred to Fåhreus slides containing PGM $\left(2.72 \mathrm{mM} \mathrm{CaCl} 2,1.95 \mathrm{mM} \mathrm{MgSO}_{4}, 2.20 \mathrm{mM}\right.$ $\mathrm{KH}_{2} \mathrm{PO}_{4}, 1.26 \mathrm{mM} \mathrm{Na}_{2} \mathrm{HPO}_{4}$, and $0.08 \mathrm{mM} \mathrm{Fe}$ (III) citrate).

Root hair deformation on $M$. truncatula is performed similarly to that on $V$. sativa, except that two plants are grown per Fåhreus slide parallel to the long side because the roots grow faster compared with $V$. sativa. Root hair deformation experiments were performed 1 day after transfer of seedlings to the slides containing PGM. For each Nod factor concentration, three slides containing two seedlings each were scored blindly (i.e., without prior knowledge of the treatment) $3 \mathrm{~h}$ after addition of the Nod factor.

\section{Fluorescence microscopy.}

Digital imaging of root hairs was performed as described (Goedhart et al. 2000), except that, in some cases, the fluorescent LCO solution was removed after $15 \mathrm{~min}$ of incubation by washing two times with PGM. For imaging whole roots, a Leica MZFLIII stereomicroscope (Leitz, Wetzlar, Germany) with 545DF10 excitation and 605DF50 emission filters was equipped with a slow scan series 200 (CH250) liquid cooled CCD camera (Photometrics, Tucson, AZ, U.S.A.), interfaced onto the Nubus slot of a Macintosh PowerPC 8100 computer and controlled using the IPlab Spectrum image-processing software (Signal Analytics, Vienna, VA, U.S.A.). To fully replace the medium surrounding the root hairs with PGM containing $10 \mathrm{nM}$ LCO-IV(BODIPY 558/568- $\mathrm{C}_{12}, \mathrm{~S}$ ), the root was washed thoroughly with PGM containing the fluorescent LCO, after which imaging was started immediately. All images were background and flat-field corrected.

\section{Chitinase activity.}

To a Fåhreus slide containing five seedlings of $V$. sativa or two seedlings of $M$. truncatula, $0.5 \mu \mathrm{M}$ fluorescent Nod factor in PGM was added. After the appropriate time, the medium was removed and extracted with 300 and $200 \mu$ of water-saturated nbutanol. The roots were washed with water twice, after which they were cut from the seedling and ground after freezing in liquid nitrogen. To the ground roots, $1 \mathrm{ml}$ of water-saturated nbutanol was added and mixed. After the addition of $4 \mathrm{ml}$ of $\mathrm{H}_{2} \mathrm{O}$, the mixture was transferred to a tube and phase separation was induced by centrifugation. After removal of the upper phase (approximately $200 \mu \mathrm{l}$ ), the lower phase was re-extracted with 200 $\mu \mathrm{l}$ of water-saturated butanol. The collected butanol phases were evaporated in a speedvac and redissolved in $20 \mu \mathrm{l}$ of n-butanol. The fluorescent products were separated by TLC according to Heidstra and associates (1994) and visualized by recording the blue-excited fluorescence on a STORM 840 (Molecular Dynamics Inc., Sunnyvale, CA, U.S.A.).

\section{FCM.}

The calibration procedure and FCM measurements were performed as described before (Goedhart et al. 2000). The laser radius (543-nm excitation) in the $x y$ plane $\left(\omega_{x y}\right)$ was calculated from the measured diffusion time $\left(\tau_{\text {diff }}\right)$ of TMR and the known diffusion constant $D$ of $2.8 \times 10^{-10} \mathrm{~m}^{2} / \mathrm{s}$ according to the following formula.

$$
\tau_{\text {diff }}=\frac{\omega_{x y}^{2}}{4 \cdot D}(1)
$$


The value of $\omega_{x y}$ differed from day to day and ranged from 240 to $290 \mathrm{~nm}$. The structural parameter $\left(\omega_{z} / \omega_{x y}\right)$ also was obtained from the measured autocorrelation curves of TMR and was between 6.4 and 9.1. For the in vivo measurements, a twocomponent fit procedure was used to obtain two diffusional times and their relative contribution (Goedhart et al. 2000). The diffusion time $\tau_{\text {diff }}$ was converted to the diffusion constant $D$ according to equation 1 , using the laser radius $\omega_{x y}$ obtained from the calibration.

\section{ACKNOWLEDGMENTS}

This work was supported by the Netherlands Organization for Scientific Research-Council of Earth and Life Sciences (NWO-ALW 805.33.344), by a NWO-Van Gogh French-Netherlands collaborative research grant (VGP 81-687), and the SACC-SIG-NET E.C. research training network (HPRN-CT-2002-00251). We thank M. A. Hink (Laboratory for Biochemistry, Wageningen University, The Netherlands) for helpful suggestions and comments on the FCM analysis; E. Dolgikh (All Russia Research Institute for Agricultural Microbiology, St. Petersburg, Russia), supported by a NATO fellowship, for experiments testing Nod factor depletion with radiolabeled Nod factors; A. Jauneau (IFR40, Pôle de Biotechnologie Végétale, Auzeville, France) for CLSM assistance; and J. Cullimore (UMR INRA-CNRS 215, Auzeville, France) and R. Ranjeva (UMR UPS-CNRS 5546, Auzeville, France) for helpful discussions and critical review of the manuscript.

\section{LITERATURE CITED}

Batzri, S., and Korn, E. D. 1973. Single bilayer liposomes prepared without sonication. Biochim. Biophys. Acta 298:1015-1019.

Bono, J.-J., Riond, J., Nicolaou, K. C., Bockovich, N. J., Estevez, V. A., Cullimore, J. V., and Ranjeva, R. 1995. Characterization of a binding site for chemically synthesized lipo-oligosaccharidic NodRm factors in particulate fractions prepared from roots. Plant J. 7:253-260.

Brock, R., Hink, M. A., and Jovin, T. M. 1998. Fluorescence correlation microscopy (FCM) of cells in the presence of autofluorescence. Biophys. J. 75:2547-2557.

Brock, R., Vàmosi, G., Vereb, G., and Jovin, T. M. 1999. Rapid characterization of green fluorescent protein fusion proteins on the molecular and cellular level by fluorescence correlation microscopy. Proc. Natl. Acad. Sci. U.S.A. 96:10123-10128.

Cullimore, J. V., Ranjeva, R., and Bono, J.-J. 2001. Perception of lipochitooligosaccharidic Nod factors in legumes. Trends Plant Sci. 6:24-30.

Demont-Caulet, N., Maillet, F., Tailler, D., Jacquinet, J. C., Prome, J. C., Nicolaou, K. C., Truchet, G., Beau, J. M., and Denarie, J. 1999. Nodule-inducing activity of synthetic Sinorhizobium meliloti nodulation factors and related lipo-chitooligosaccharides on alfalfa. Importance of the acyl chain structure. Plant Physiol. 120:83-92.

Den Hartog, M., Musgrave, A., and Munnik, T. 2001. Nod factor-induced phosphatidic acid and diacylglycerol pyrophosphate formation: a role for phospholipase $\mathrm{C}$ and $\mathrm{D}$ in root hair deformation. Plant J. 25:55-65.

de Wit, P. J., Lauge, R., Honee, G., Joosten, M. H., Vossen, P., KoomanGersmann, M., Vogelsang, R., and Vervoort, J. J. 1997. Molecular and biochemical basis of the interaction between tomato and its fungal pathogen Cladosporium fulvum. Antonie Leeuwenhoek 71:137-141.

Downie, J. A., and Walker, S. A. 1999. Plant responses to nodulation factors. Curr. Opin. Plant Biol. 2:483-489.

Ehrhardt, D. W., Wais, R., and Long, S. R. 1996. Calcium spiking in plant root hairs responding to Rhizobium nodulation signals. Cell 85:673-681.

Endre, G., Kereszt, A., Kevei, Z., Mihacea, S., Kalo, P., and Kiss, G. B. 2002. A receptor kinase gene regulating symbiotic nodule development. Nature 417:962-966.

Etzler, M. E., Kalsi, G., Ewing, N. N., Roberts, N. J., Day, R. B., and Murphy, J. B. 1999. A nod factor binding lectin with apyrase activity from legume roots. Proc. Natl. Acad. Sci. U.S.A. 96:5856-5861.

Felle, H. H., Kondorosi, E., Kondorosi, A., and Schultze, M. 1998. The role of ion fluxes in Nod factor signaling in Medicago sativa. Plant $\mathrm{J}$. 13:455-465.

Gadella, T. W. J., Jr., Vereb, G., Hadri, A.-E., Röhrig, H., Schmidt, J., John, M., Schell, J., and Bisseling, T. 1997. Microspectroscopic imaging of nodulation factor-binding sites on living Vicia sativa roots using a novel bioactive fluorescent nodulation factor. Biophys. J. 72:1986-1996.

Goedhart, J., Hink, M. A., Visser, A. J., Bisseling, T., and Gadella, T. W. J., Jr. 2000. In vivo fluorescence correlation microscopy (FCM) re- veals accumulation and immobilization of Nod factors in root hair cell walls. Plant J. 21:109-119.

Goedhart, J., Rohrig, H., Hink, M. A., van Hoek, A., Visser, A. J., Bisseling, T., and Gadella, T. W. J. 1999. Nod factors integrate spontaneously in biomembranes and transfer rapidly between membranes and to root hairs, but transbilayer flip-flop does not occur. Biochemistry 38:10898-10907.

Gressent, F., Drouillard, S., Mantegazza, N., Samain, E., Geremia, R. A., Canut, H., Niebel, A., Driguez, H., Ranjeva, R., Cullimore, J., and Bono, J.-J. 1999. Ligand specificity of a high-affinity binding site for lipo-chitooligosaccharidic nod factors in Medicago cell suspension cultures. Proc. Natl. Acad. Sci. U.S.A. 96:4704-4709.

Heidstra, R., and Bisseling, T. 1996. Nod factor-induced host responses and mechanisms of Nod factor perception. New Phytol. 133:25-43.

Heidstra, R., Geurts, R., Franssen, H., Spaink, H., van Kammen, A., and Bisseling, T. 1994. Root hair deformation activity of nodulation factors and their fate on Vicia sativa. Plant Physiol. 105:787-797.

Hess, S. T., Huang, S., Heikal, A .A., and Webb, W. W. 2002. Biological and chemical applications of fluorescence correlation spectroscopy: A review. Biochemistry 41:697-705.

Hovius, R., Vallotton, P., Wohland, T., and Vogel, H. 2000. Fluorescence techniques: shedding light on ligand-receptor interactions. Trends Pharmacol. Sci. 21:266-273.

Lerouge, F., Roche, P., Faucher, C., Maillet, F., Truchet, G., Promé, J. C., and Dénarié, J. 1990. Symbiotic host-specificity of Rhizobium meliloti is determined by a sulphated and acylated glucosamine oligosaccharide signal. Nature 344:781-784

Long, S. R. 1996. Rhizobium symbiosis: Nod factors in perspective. Plant Cell 8:1885-1898.

Maiti, S., Haupts, U., and Webb, W. W. 1997. Fluorescence correlation spectroscopy: diagnostics for sparse molecules. Proc. Natl. Acad. Sci. U.S.A. 94:11753-11757.

Niebel, A., Bono, J.-J., Ranjeva, R., and Cullimore, J. V. 1997. Identification of a high affinity binding site for lipooligosaccharidic NodRm factors in the microsomal fraction of Medicago cell suspension cultures. Mol. Plant-Microbe Interact. 10:132-134.

Oldroyd, G. E., Mitra, R. M., Wais, R. J., and Long, S. R. 2001. Evidence for structurally specific negative feedback in the Nod factor signal transduction pathway. Plant J. 28:191-199.

Pingret, J. L., Journet, E. P., and Barker, D. G. 1998. Rhizobium nod factor signaling. Evidence for a g protein-mediated transduction mechanism. Plant Cell 10:659-672.

Roche, P., Debelle, F., Maillet, F., Lerouge, P., Faucher, C., Truchet, G., Denarie, J., and Prome, J. C. 1991. Molecular basis of symbiotic host specificity in Rhizobium meliloti: nodH and nodPQ genes encode the sulfation of lipo-oligosaccharide signals. Cell 67:1131-1143.

Schwille, P., Haupts, U., Maiti, S., and Webb, W. W. 1999. Molecular dynamics in living cells observed by fluorescence correlation spectroscopy with one- and two-photon excitation. Biophys. J. 77:2251-2265.

Shechter, Y., Schlessinger, J., Jacobs, S., Chang, K. J., and Cuatrecasas, P. 1978. Fluorescent labeling of hormone receptors in viable cells: preparation and properties of highly fluorescent derivatives of epidermal growth factor and insulin. Proc. Natl. Acad. Sci. U.S.A. 75:2135-2139.

Spaink, H. P., Sheely, D. M., Van Brussel, A. A. N., Glushka, J., York, W. S., Tak, T., Geiger, O., Kennedy, E. P., Reinhold, V. N., and Lugtenberg, B. J. J. 1991. A novel highly unsaturated fatty acid moiety of lipo-oligosaccharide signals determines host specificity in Rhizobium. Nature 354:125-130

Staehelin, C., Schultze, M., Kondorosi, E., Mellor, R. B., Boller, T., and Kondorosi, A. 1994. Structural modifications in Rhizobium melilot Nod factors influence their stability against hydrolysis by root chitinases. Plant J. 5:319-330.

Stracke, S., Kistner, C., Yoshida, S., Mulder, L., Sato, S., Kaneko, T., Tabata, S., Sandal, N., Stougaard, J., Szczyglowski, K., and Parniske, M. 2002. A plant receptor-like kinase required for both bacterial and fungal symbiosis. Nature 417:959-962.

Van der Holst, P. P. G., Schlaman, H. R. M., and Spaink, H. P. 2001. Proteins involved in the production and perception of oligosaccharides in relation to plant and animal development. Curr. Opin. Struct. Biol. 11:608-616.

Wais, R. J., Galera, C., Oldroyd, G., Catoira, R., Penmetsa, R. V., Cook, D., Gough, C., Denarie, J., and Long, S. R. 2000. Genetic analysis of calcium spiking responses in nodulation mutants of Medicago truncatula. Proc. Natl. Acad. Sci. U.S.A. 97:13407-13412.

Walker, S. A., Viprey, V., and Downie, J. A. 2000. Dissection of nodulation signaling using pea mutants defective for calcium spiking induced by nod factors and chitin oligomers. Proc. Natl. Acad. Sci. U.S.A. 97:13413-13418. 